\title{
Role of Time Relaxation in a One-Dimensional Diffusion-Advection Model of Water and Salt Transport
}

\author{
Igor Medved'1,2 and Robert Černý ${ }^{1}$ \\ ${ }^{1}$ Department of Materials Engineering and Chemistry, Faculty of Civil Engineering, Czech Technical University in Prague, \\ Thakurova 7, 16629 Prague, Czech Republic \\ ${ }^{2}$ Department of Physics, Constantine the Philosopher University, 94974 Nitra, Slovakia
}

Correspondence should be addressed to Igor Medved'; imedved@ukf.sk

Received 6 September 2015; Revised 2 November 2015; Accepted 8 November 2015

Academic Editor: Yao-Zhong Zhang

Copyright (C) 2015 I. Medved' and R. Černý. This is an open access article distributed under the Creative Commons Attribution License, which permits unrestricted use, distribution, and reproduction in any medium, provided the original work is properly cited.

\begin{abstract}
The transport of salt, necessarily coupled with the transport of water, through porous building materials may heavily limit their durability due to possible deterioration and structural damage. Usually, the binding of salt to the pore walls is assumed to occur instantly, as soon as the salt is transported by water to a given position. We consider the advection-diffusion model of the transport and generalize it to include possible delays in the binding. Applying the Boltzmann-Matano method, we calculate the diffusion coefficient of the salt in dependence on the salt concentration and show that it increases with the rate of binding. We apply our results to an example of the chloride transport in a lime plaster.
\end{abstract}

\section{Introduction}

One of the key problems in real situations where the mass transport by diffusion occurs is the fact that the corresponding diffusion coefficients may strongly depend on the concentration of the transported species. Without a detailed knowledge of this dependence, the analysis of the transport could be rather inaccurate and yield misleading results. If the transport is simple enough to be described as onedimensional, then the Boltzmann-Matano method $[1,2]$ is often applied as a convenient technique to derive a varying diffusion coefficient. To do so, the method uses a known concentration profile (usually from experimental data), that is, the concentration as a function of the Boltzmann variable $\eta=x / \sqrt{t}$, where $x$ and $t$ are the position and time, respectively.

The calculation of the diffusion coefficient by the Boltzmann-Matano method is most frequently performed numerically. This has several drawbacks: the resulting diffusion coefficient is given in a discrete format and may require a final smoothing to remove illogical jumps or spikes, the reliability of the results should be verified by back-calculation of the original profile, and the sensitivity of the results to various model parameters is rather time consuming to examine [3]. In addition, the whole numerical determination procedure must be performed afresh for each studied situation. It is possible to avoid all of these drawbacks by employing an analytical approach. For such a case, the concentration profile is approximated by an analytical curve from which a formula for the varying diffusion coefficient is obtained [4-8].

The analytical approach can be a very useful tool for practical engineering calculations. We applied the approach in [9] to the diffusion-advection model of Bear and Bachmat [10] that has been used to study a coupled water and salt transport in porous building materials. In this case, proper understanding of the transport is crucial for the assessment of durability of the materials, because moisture and salt can cause serious deterioration and structural damage. In [9] it was implicitly assumed that the binding of salt to the pore walls occurs instantly whenever the salt is transported by water to a given position. Here we wish to generalize our analytical results to include the fact that the binding of salt is somewhat delayed and to study the effect of this phenomenon on the diffusion coefficient of salt. Such an effect has been lately studied in connection with the chloride ion penetration into concrete, using a transport model based on the concrete 
electrical resistivity [11]. They accounted for a slower chloride advance simply by adding a phenomenological multiplication factor in the apparent diffusion coefficient. In this study our approach will be more advanced: we implement the relaxation into the salt concentration profile and examine how this affects the diffusion coefficient.

\section{Theoretical Background}

2.1. The Diffusion-Advection Model. The Bear-Bachmat diffusion-advection model for the transport of a salt solution together with the water mass balance is given as [10]

$$
\begin{aligned}
\frac{\partial\left(w C_{f}\right)}{\partial t} & =\nabla \cdot\left[w D\left(C_{f}\right) \nabla C_{f}\right]-\nabla \cdot\left(C_{f} \mathbf{v}\right)-\frac{\partial C_{b}}{\partial t}, \\
\frac{\partial w}{\partial t} & =-\nabla \cdot \mathbf{v}
\end{aligned}
$$

where $w$ is the volumetric moisture content, $C_{f}$ is the concentration of a free salt in water, $C_{b}$ is the concentration of a bound salt in the whole porous body, $D$ is the salt diffusion coefficient, and $\mathbf{v}$ is the Darcy velocity. Since $\mathbf{v}$ can be expressed via the moisture content as $\mathbf{v}=-\kappa(w) \nabla w$, where $\kappa$ is the moisture diffusivity, model (1) is a system of two coupled partial differential equations with two material parameters, $\kappa$ and $D$, and three input quantities, $w, C_{f}$, and $C_{b}$. Measurable quantities are the moisture content $w$ and the total concentration, $C$, given as

$$
C=w C_{f}+C_{b}
$$

Nevertheless, all three input quantities are known, as soon as the binding isotherm, that is, the dependence

$$
C_{b}=F\left(C_{f}\right)
$$

is determined for the studied material from an experiment.

In the one-dimensional case, model (1) may be rewritten as

$$
\begin{aligned}
& \frac{\partial C}{\partial t}=\frac{\partial}{\partial x}\left[w D\left(C_{f}\right) \frac{\partial C_{f}}{\partial x}+C_{f} \kappa(w) \frac{\partial w}{\partial x}\right], \\
& \frac{\partial w}{\partial t}=\frac{\partial}{\partial x}\left[\kappa(w) \frac{\partial w}{\partial x}\right] .
\end{aligned}
$$

Here $C_{f}=C_{f}(w, C)$ is the solution of the equation $C_{f}=(C-$ $\left.F\left(C_{f}\right)\right) / w$ (see (2) and (3)). In formulation (4) of the model there are two quantities, $w(x, t)$ and $C(x, t)$, to be found.

In a typical experiment, a completely dry prismatic sample is exposed by one of its faces to a penetrating salt solution (water) with a concentration $C_{0}$ (with a moisture content $w_{0}$ ). At the same time, the opposite face is kept dry, while the lateral sides are insulated to make the solution transport one-dimensional. The initial and boundary conditions corresponding to such an experiment with an idealized infinitely long sample are given as

$$
\begin{aligned}
C(0, t) & =C_{0}, \\
C(\infty, t) & =C(x, 0)=0, \\
w(0, t) & =w_{0}, \\
w(\infty, t) & =w(x, 0)=0 .
\end{aligned}
$$

2.2. Relaxation in Binding of Salt. In the binding isotherm (3) the salt is assumed to become bound at a given position, $x$, immediately at the time when the salt is first transported by water flow to $x$. Note that this time depends on position $x$. In reality, however, the binding is always somewhat delayed. We may take this fact into account by replacing isotherm (3) by the relation

$$
C_{b}(x, t)=g(x, t) F\left(C_{f}(x, t)\right),
$$

where $g(x, t)$ characterizes the delay of the salt binding. It should be vanishing before the time, $t_{x}$, when the salt is first transported by water to a position $x$. In addition, it should be increasing after $t=t_{x}$, approaching 1 as $t \rightarrow \infty$. We will consider a simple form of $g$ given as follows (see Figure 1(a)):

$$
g(x, t)= \begin{cases}0 & 0 \leq t \leq t_{x}, \\ 1-\exp \left(-\frac{t-t_{x}}{\tau}\right) & t \geq t_{x},\end{cases}
$$

in which the onset of the salt binding (which begins at $t_{x}$ ) is described by the exponential with a relaxation time $\tau>0$ (see Figure 1). As $\tau$ approaches zero, $g$ turns into a step function, which corresponds to an immediate binding of salt. Thus, in the limit $\tau \rightarrow 0$, the modified isotherm (6) yields the original isotherm (3) (note that $C_{b}=0$ at any position $x$ for $t<t_{x}$ ). On the other hand, the limit $\tau \rightarrow \infty$ yields a vanishing $g$, which corresponds to no binding of salt.

In many experiments the involved concentrations are low, so that the linear (Henry) isotherm is applicable; $F\left(C_{f}\right)=$ $K_{H} C_{f}$ with a constant $K_{H}>0$. We will consider this isotherm in the following. Then the free concentration in the onedimensional model (4) is explicitly given as

$$
C_{f}(x, t)=\frac{C(x, t)}{w(x, t)+K_{H} g(x, t)} .
$$

2.3. The Boltzmann-Matano Method. Using the transformation $\eta=x / \sqrt{t}$ and observing that $\partial / \partial x=(1 / \sqrt{t}) d / d \eta$ and $\partial / \partial t=-(\eta / 2 t) d / d \eta$, the one-dimensional diffusionadvection model (4) may be rewritten as

$$
\begin{aligned}
& -\eta w^{\prime}=2\left(\kappa w^{\prime}\right)^{\prime}, \\
& -\eta C^{\prime}=2\left(w D C_{f}^{\prime}+C_{f} \kappa w^{\prime}\right)^{\prime},
\end{aligned}
$$




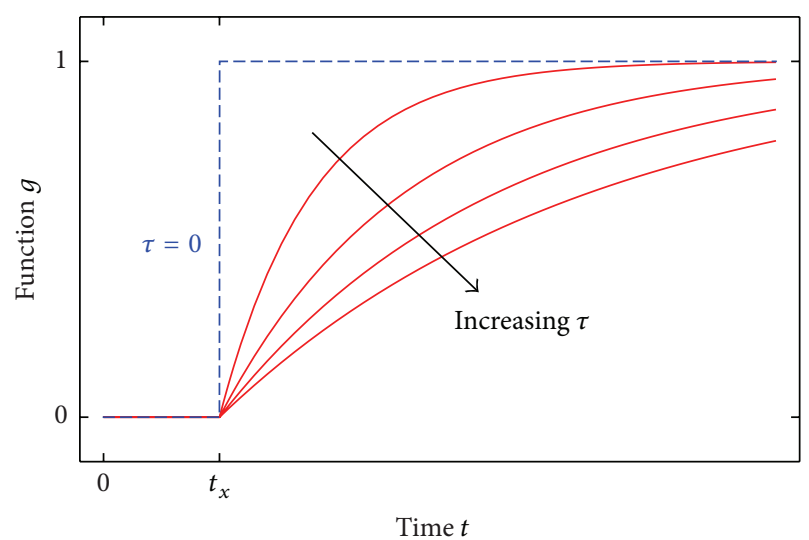

(a)

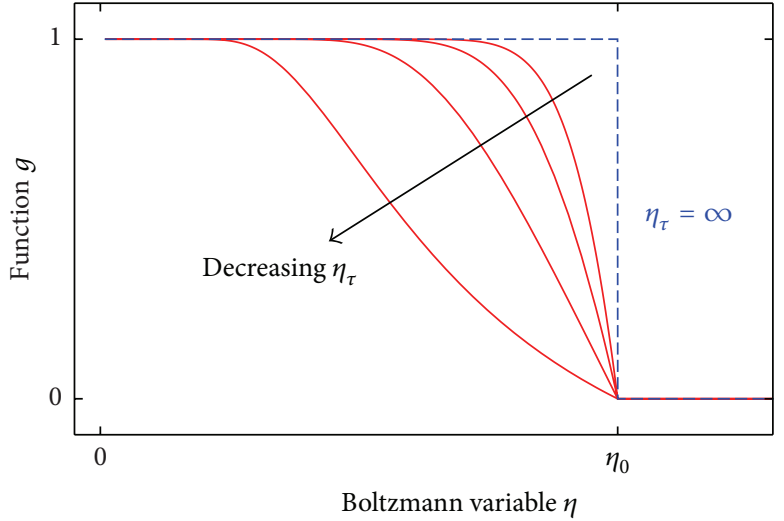

(b)

Figure 1: (a) The function $g(x, t)$ at a fixed position $x$ for several values of the relaxation time $\tau$ (the full lines). The step-wise form (the dashed line) corresponds to an immediate binding of salt $(\tau=0)$. (b) The corresponding function $g(\eta)$.

where the prime indicates the derivative with respect to $\eta$ (called the Boltzmann variable). The free concentration from (8) is here given as $C_{f}(\eta)=C(\eta) /\left[w(\eta)+K_{H} g(\eta)\right]$ with

$$
g(\eta)= \begin{cases}1-\exp \left[-\eta_{\tau}^{2}\left(\frac{1}{\eta^{2}}-\frac{1}{\eta_{0}^{2}}\right)\right] & 0 \leq \eta \leq \eta_{0}, \\ 0 & \eta \geq \eta_{0},\end{cases}
$$

where $\eta_{0}=x / \sqrt{t_{x}}$ (above this value the concentration profile $C(\eta)$ is negligible) and $\eta_{\tau}=x / \sqrt{\tau}$ (see Figure 1(b)). The initial and boundary conditions $(5)$ read $C(\eta=0)=C_{0}, w(\eta=0)=$ $w_{0}$, and $C(\eta=\infty)=w(\eta=\infty)=0$.

We may integrate (9) from $\eta$ to $\infty$, assuming that both $w^{\prime}(\eta)$ and $C^{\prime}{ }_{f}(\eta)$ vanish as $\eta \rightarrow \infty$ (because both $w$ and $C$ approach constants at large $x$ ). This will enable us to express the moisture diffusivity and diffusion coefficient as $[12,13]$

$$
\begin{aligned}
\kappa(\eta) & =\frac{I_{w}(\eta)}{2 w^{\prime}(\eta)}, \\
D(\eta) & =-\frac{C_{f}(\eta) I_{w}(\eta)}{2 w(\eta) C^{\prime}{ }_{f}(\eta)}+\frac{I_{C}(\eta)}{2 w(\eta) C^{\prime}{ }_{f}(\eta)} \\
& \equiv D_{1}(\eta)+D_{2}(\eta) .
\end{aligned}
$$

Here we expressed $D$ as the sum of two contributions, $D_{1}$ and $\mathrm{D}_{2}$, and we introduced the integrals

$$
\begin{aligned}
& I_{w}(\eta) \equiv \int_{\eta}^{\infty} \eta^{*} w^{\prime}\left(\eta^{*}\right) d \eta^{*}, \\
& I_{C}(\eta) \equiv \int_{\eta}^{\infty} \eta^{*} C^{\prime}\left(\eta^{*}\right) d \eta^{*} .
\end{aligned}
$$

Thus, the moisture diffusivity $\kappa$ and the diffusion coefficient $D$ can be calculated from (11) and (12), provided the moisture and concentration profiles $w(\eta)$ and $C(\eta)$ are known. Note that $I_{w}<0, I_{C}<0, \kappa>0, D_{2}>0$, and $D_{1}<0$, because the moisture and concentration profiles are decreasing (as follows from the initial and boundary conditions in (5)). Thus, to get a positive diffusion coefficient $D$, it is necessary that $\left|D_{1}\right|$ is smaller than $\mathrm{D}_{2}$.

2.4. Analytical Approach: Moisture Diffusivity. It may be difficult to numerically evaluate the moisture diffusivity $\kappa$ and diffusion coefficient $D$ from (11) and (12), respectively, using experimental data on the profiles $w(\eta)$ and $C(\eta)$. This is mainly due to possible inaccuracies in the evaluation of the derivatives $w^{\prime}$ and $C_{f}^{\prime}$. An alternative approach is, therefore, to use model formulas to accurately approximate the profiles and subsequently evaluate $\kappa$ and $D$ analytically. This approach goes back to the 1953 work of Hall [4] and has been conveniently used lately [5-9].

Similarly to $[4,9]$, we will use here the error function, erf, as the model formula to approximate the profiles. This choice is expected to be reasonable when the experimentally measured profiles are "S-shaped." It is inspired by the fact that the error function is known to be an exact solution to the onedimensional diffusion equation with a constant diffusivity $[14,15]$.

For the moisture profile we will consider the model profile

$$
\mathrm{w}(\eta) \equiv h_{1} \operatorname{erfc}\left[\frac{\sqrt{\pi}}{2} a_{1}\left(\eta-\eta_{1}\right)\right],
$$

where erf $\mathrm{c}=1-$ erf is the complementary error function. The parameters $a_{1}$ and $\eta_{1}$ specify the slope at the profile's maximal drop and its position, respectively, and $h_{1}=w_{0} / 2$ is the halfheight of the moisture profile. The factor $\sqrt{\pi} / 2$ was added just to make the slope equal to $-a_{1} h_{1}$ rather than to $-2 a_{1} h_{1} / \sqrt{\pi}$. If one approximates the experimental moisture profile by model one, $w(\eta) \approx \mathrm{w}(\eta)$, from (11) it follows that [9]

$$
I_{w}(\eta) \approx \frac{2 w^{\prime}(\eta)}{\pi a_{1}^{2}}-\eta_{1} w(\eta)
$$

yielding

$$
\kappa(\eta)=\frac{1}{\pi a_{1}^{2}}+\frac{\eta_{1}}{2 a_{1}} \operatorname{erfc}\left[\frac{\sqrt{\pi}}{2} a_{1}\left(\eta-\eta_{1}\right)\right] e^{\pi a_{1}^{2}\left(\eta-\eta_{1}\right)^{2} / 4} .
$$


Using also (14), one may express the moisture diffusivity as a function of the moisture content [9]:

$$
\kappa(w) \approx \frac{1}{\pi a_{1}^{2}}+\frac{\eta_{1}}{2 a_{1}} y e^{E^{2}} \equiv \kappa_{0}+\kappa_{1}(w),
$$

where $y \equiv 2 w / w_{0}$ and $E \equiv \operatorname{erfc}^{-1} y$. We use $\operatorname{erfc}^{-1}$ to denote the inverse complementary error function. Expression (17) for the moisture dependence of the moisture diffusivity is in agreement with the general result derived already by Hall [4]. Note that, at low moisture contents, $\kappa(w) \approx \kappa_{0}+$ $\eta_{1} / 2 a_{1}(\pi|\ln y|)^{1 / 2}$, so that the moisture diffusivity slowly approaches the constant value $\kappa_{0}$ as $w$ vanishes.

2.5. Analytical Approach: Diffusion Coefficient. If we employ a model profile for the total concentration that is similar to the moisture profile,

$$
\mathrm{C}(\eta) \equiv h_{2} \operatorname{erfc}\left[\frac{\sqrt{\pi}}{2} a_{2}\left(\eta-\eta_{2}\right)\right],
$$

where $h_{2}=C_{0} / 2, a_{2}$, and $\eta_{2}$ have an analogous meaning as in (14), we can obtain a formula for the $\eta$ dependence of the diffusion coefficient $D$. Indeed, in the approximations $w(\eta) \approx$ $\mathrm{w}(\eta)$ and $C(\eta) \approx \mathrm{C}(\eta)$, the free salt concentration is given as

$$
\begin{aligned}
C_{f}(\eta) & \approx \lambda(\eta), \\
C^{\prime}{ }_{f}(\eta) & \approx \lambda(\eta) q(\eta),
\end{aligned}
$$

where

$$
\begin{aligned}
& \lambda(\eta) \equiv \frac{\mathrm{C}(\eta)}{\mathrm{w}(\eta)+K_{H} g(\eta)} \\
& q(\eta) \equiv \frac{\mathrm{C}^{\prime}(\eta)}{\mathrm{C}(\eta)}-\frac{\mathrm{w}^{\prime}(\eta)+K_{H} g^{\prime}(\eta)}{\mathrm{w}(\eta)+K_{H} g(\eta)}
\end{aligned}
$$

with

$$
\begin{aligned}
& \mathrm{w}^{\prime}(\eta)=-a_{1} h_{1} e^{(-\pi / 4) a_{1}^{2}\left(\eta-\eta_{1}\right)^{2}}, \\
& \mathrm{C}^{\prime}(\eta)=-a_{2} h_{2} e^{(-\pi / 4) a_{2}^{2}\left(\eta-\eta_{2}\right)^{2}} .
\end{aligned}
$$

Since the integral

$$
I_{C}(\eta) \approx \frac{2 C^{\prime}(\eta)}{\pi a_{2}^{2}}-\eta_{2} \mathrm{C}(\eta),
$$

analogously to (15), from (12), we get

$$
\begin{aligned}
D(\eta)= & D_{1}(\eta)+D_{2}(\eta) \\
\approx & -\frac{1}{2 q(\eta)}\left[\frac{2 \mathrm{w}^{\prime}(\eta)}{\pi a_{1}^{2} \mathrm{w}(\eta)}-\eta_{1}\right] \\
& +\frac{1}{2 q(\eta)}\left[\frac{2 \mathrm{C}^{\prime}(\eta)}{\pi a_{2}^{2} \mathrm{C}(\eta)}-\eta_{2}\right] \frac{\mathrm{C}(\eta)}{\lambda(\eta) \mathrm{w}(\eta)} .
\end{aligned}
$$

This is the desired expression for the $\eta$ dependence of the diffusion coefficient. Its explicit form-which follows upon plugging in the formulas from (14), (18), (20), and (21)-is rather complex.
TABLE 1: The best-fit values of the profiles' parameters for the erfc approximations. The model profile fits are in good agreement with the data: the coefficient of determination and root mean square error are $R^{2}=0.9972$ and RMSE $=8.64 \times 10^{-3}$ for the moisture profile and $R^{2}=0.9949$ and $\mathrm{RMSE}=0.152 \mathrm{~kg} \cdot \mathrm{m}^{-3}$ for the concentration profile [9].

\begin{tabular}{lcccc}
\hline $\begin{array}{l}\text { Moisture content } \\
\text { profile }\end{array}$ & $\begin{array}{c}w_{0} \\
(-)\end{array}$ & $\begin{array}{c}h_{1} \\
(-)\end{array}$ & $\begin{array}{c}a_{1} \\
\left(\mathrm{~m}^{-1} \mathrm{~s}^{1 / 2}\right)\end{array}$ & $\begin{array}{c}\eta_{1} \\
\left(\mathrm{~m} \cdot \mathrm{s}^{-1 / 2}\right)\end{array}$ \\
\hline $\begin{array}{l}\text { Total chloride } \\
\text { concentration }\end{array}$ & $\begin{array}{c}C_{0} \\
\text { profile }\end{array}$ & $\begin{array}{c}h_{2} \\
\left.\mathrm{~kg} \cdot \mathrm{m}^{-3}\right)\end{array}$ & $\begin{array}{c}\mathrm{k}_{2} \\
\left.\mathrm{~kg} \cdot \mathrm{m}^{-3}\right)\end{array}$ & $\begin{array}{c}\left.\mathrm{m}^{-1} \mathrm{~s}^{1 / 2}\right) \\
\left(\mathrm{m} \cdot \mathrm{s}^{-1 / 2}\right)\end{array}$ \\
\hline
\end{tabular}

\section{Application to Experiment}

Let us illustrate our above results and obtain the moisture diffusivity and diffusion coefficient for an experiment in which a one-dimensional coupled moisture and chloride transport in a lime plaster was studied [13] (see the paper for the experimental details). The corresponding profiles $w(\eta)$ and $C(\eta)$ are shown in Figure 2. The figure also shows the least-square fits of the erfc model profiles (14) and (18) to the experimental data. The fitting parameters are listed in Table 1. Note that $\eta_{0} \approx 9.5 \times 10^{-4} \mathrm{~m} \mathrm{~s}^{-1 / 2}$ in this experiment.

In addition, the chloride binding isotherm for the studied lime plaster turns out to be rather precisely linear: up to $C_{f}=36 \mathrm{~kg} \cdot \mathrm{m}^{-3}$, the line $C_{b}=K_{H} C_{f}$ with the constant $K_{H}=2.43$ fits the experimental data with good accuracy [9]. It can be therefore safely assumed that the Henry binding isotherm is applicable. Using this fact and (8), the free chloride concentration is shown in Figure 3(a). The profile strongly depends on the value of $\eta_{\tau}$ that specifies how fast the chloride binding in the lime plaster occurs. We will exclude small values $0 \leq \eta_{\tau} \leq 0.9 \eta_{0}$ (i.e., too slow binding of salt) for which the profile is physically inappropriate (not decreasing). We will discard the part of the profile within the interval $\eta>\eta_{0}$ for the same reason. The so-obtained concentration profiles $C_{f}(\eta)$ are plotted in Figure 3(b).

Applying (23), we obtained the $\eta$ dependence of the diffusion coefficient $D$ for several values of $\eta_{\tau}$ (see Figure 4 ). It is clear that $D$ decreases with $\eta_{\tau}$, especially at large values of $\eta$, reaching a minimum for the limiting case $\eta_{\tau} \rightarrow \infty$ when the salt binding occurs instantly. Thus, the relaxation of salt binding makes the diffusion coefficient grow. The figure also shows that the part $D_{1}$ of $D$ is negative and its absolute value does not exceed $1 / 10$ of the part $D_{2}$ (except at $\eta$ close to $\eta_{0}$ ).

If we wish to obtain the dependence of the diffusion coefficient $D$ on the free concentration $C_{f}$, we may numerically eliminate $\eta$ between the first relation in (19) and expression (23). The result is plotted in Figure 5 for the same values of $\eta_{\tau}$ as in Figure 4 . The conclusions on the behavior of $D(\eta)$ remain true also for $D\left(C_{f}\right)$. Thus, $D$ decreases as the salt binding becomes faster and faster, and the smallest $D$ corresponds to an instant salt binding. Moreover, $D_{1}$ is negative, and $\left|D_{1}\right|$ is less than a tenth of $D_{2}$.

Finally, let us note that the role of the relaxation of the salt binding is formally included in the quantities $\lambda$ and $q$. 


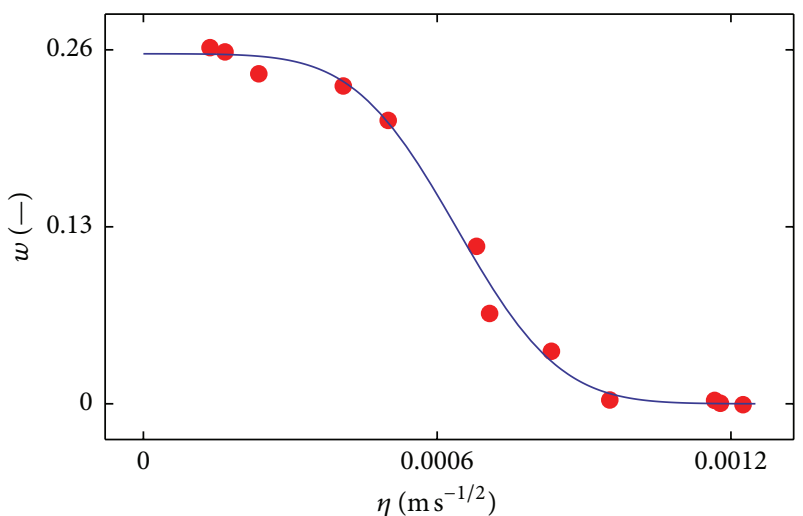

(a)

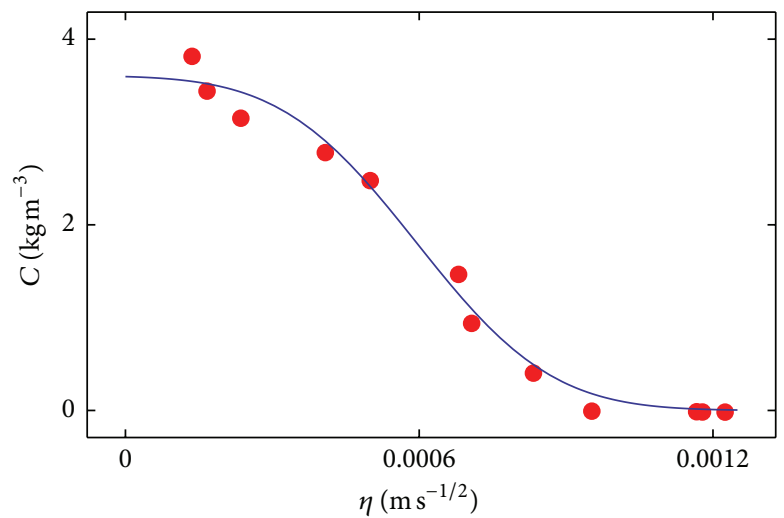

(b)

Figure 2: The profile of (a) the moisture content and (b) the total chloride concentration obtained experimentally (disks) and their approximations by the erfc model profiles (curves), as adapted from [9].

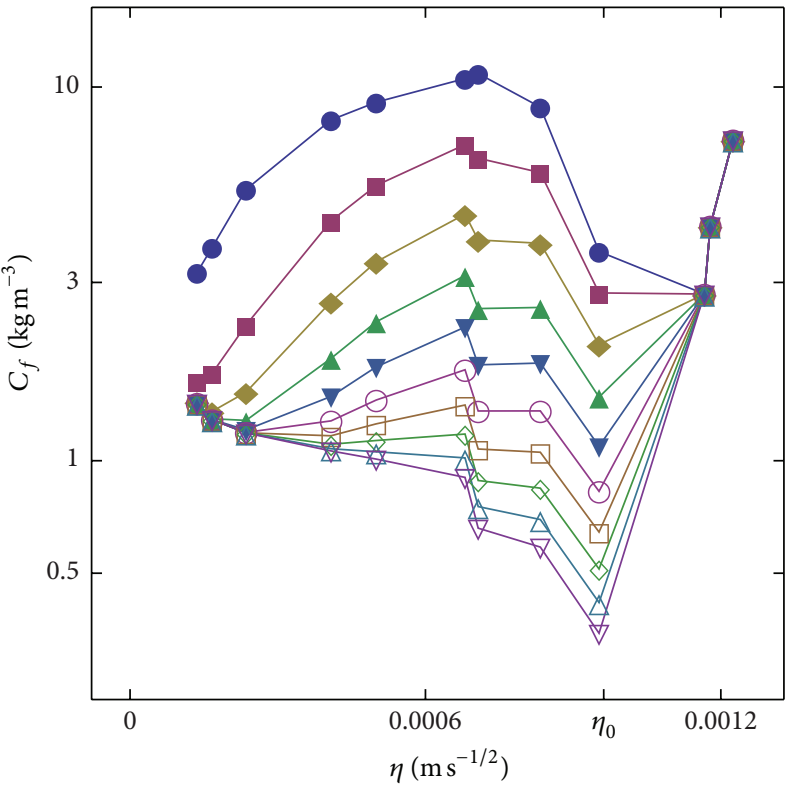

(a)

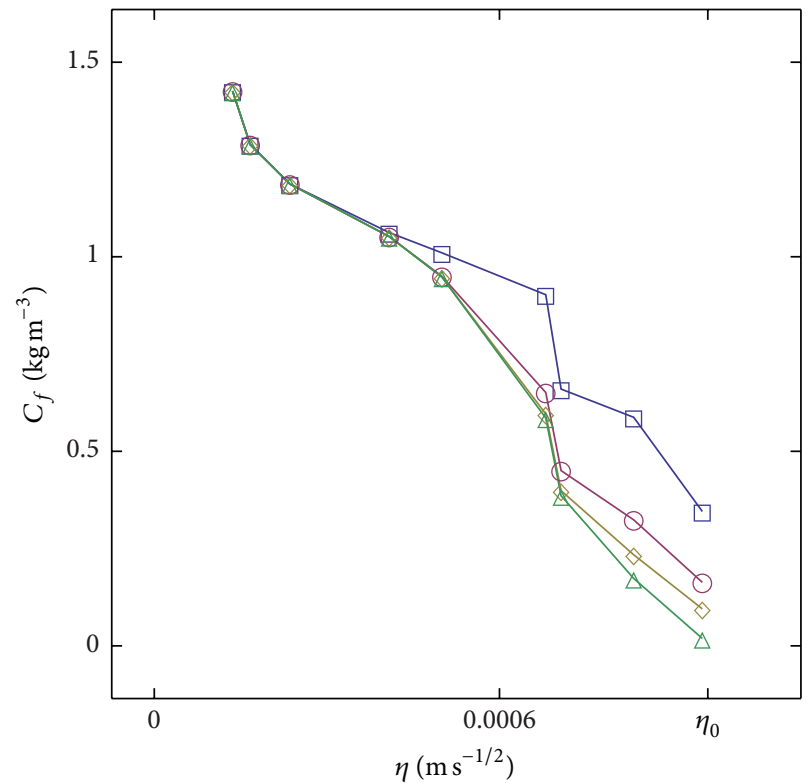

(b)

Figure 3: (a) The profile of the free chloride concentration obtained from the experimental data for $\eta_{\tau}=0.1 \eta_{0}, 0.2 \eta_{0}, \ldots, \eta_{0}$ (from top to bottom). (b) The profile of the free chloride concentration in the interval $\eta \leq \eta_{0}$ for $\eta_{\tau}=\eta_{0}, 1.5 \eta_{0}, 2 \eta_{0}, 5 \eta_{0}$ (from top to bottom).

The reason is that only these quantities contain the function $g$ that simulates the delay in the salt binding. When there is no delay $(g=1)$, the expression (23) for $D(\eta)$ reduces to the corresponding expression obtained in [9] where instant salt binding was considered. The present results are thus generalizations of those from [9].

\section{Conclusions}

We considered the advection-diffusion model of a coupled water and salt transport in porous materials in which the time relaxation of the binding of salt was included. We applied the Boltzmann-Matano method to calculate the diffusion coefficient of the salt in dependence on the salt concentration, using model profiles for the moisture and concentration expressed via the error function. We used the resulting formulas to study, as an example, a coupled moisture and chloride transport in a lime plaster. It was shown that the chloride diffusion coefficient in this experiment increases with the rate of chloride binding. Its minimal value corresponded to an instant binding, which is implicitly assumed in most models of salt transport.

\section{Conflict of Interests}

The authors declare that there is no conflict of interests regarding the publication of this paper. 


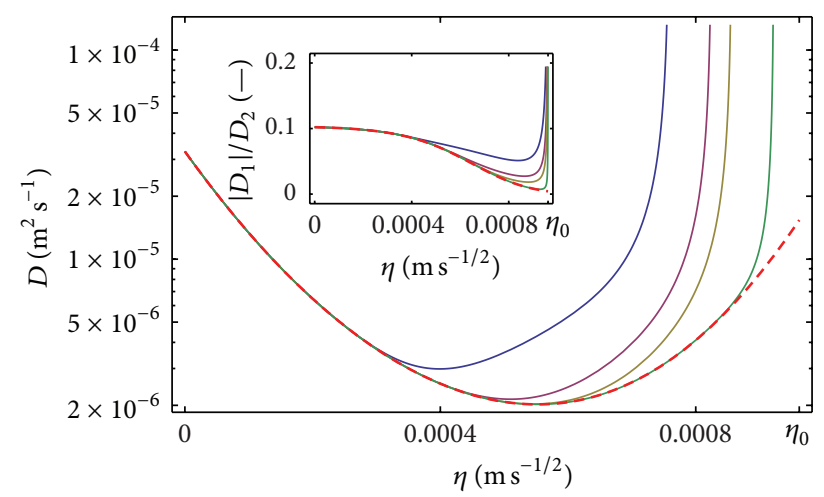

FIgURE 4: The dependence of the chloride diffusion coefficient on the Boltzmann variable. The full lines correspond to formula (23), using $\eta_{\tau}=\eta_{0}, 1.5 \eta_{0}, 2 \eta_{0}, 5 \eta_{0}$ (from left to right). The inset shows that the relative contribution of $D_{1}$ to $D$ is quite small, not exceeding $10 \%$. The dashed line indicates the case with an immediate binding of salt $\left(\eta_{\tau} \rightarrow \infty\right)$.

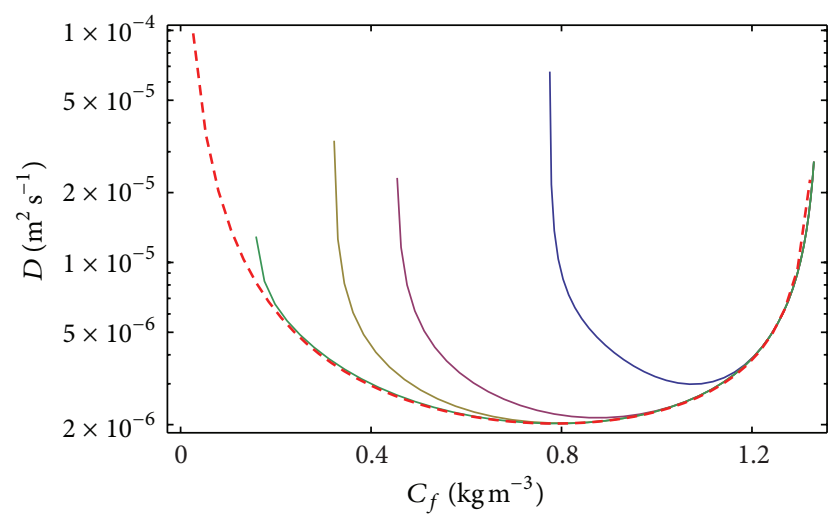

FIGURE 5: The concentration dependence of the chloride diffusion coefficient obtained numerically (the full lines) for $\eta_{\tau}=$ $\eta_{0}, 1.5 \eta_{0}, 2 \eta_{0}, 5 \eta_{0}$ (from right to left). The thick dashed line indicates the case with an immediate binding of salt $\left(\eta_{\tau} \rightarrow \infty\right)$.

\section{Acknowledgment}

This research was supported by the Czech Science Foundation under Project no. P105/12/G059.

\section{References}

[1] L. Boltzmann, "Zur integration des diffusiongleichung bei variabeln diffusions coefficienten," Annals of Physics, vol. 53, p. 959, 1894.

[2] C. Matano, "On the relation between the diffusion-coefficients and concentrations of solid metals (the nickel-copper system)," Japanese Journal of Physics, vol. 8, no. 3, pp. 109-113, 1933.

[3] J. Carmeliet, H. Hens, S. Roels et al., "Determination of the liquid water diffusivity from transient moisture transfer experiments," Journal of Thermal Envelope and Building Science, vol. 27, no. 4, pp. 277-305, 2004.

[4] L. D. Hall, "An analytical method of calculating variable diffusion coefficients," The Journal of Chemical Physics, vol. 21, no. 1, pp. 87-89, 1953.
[5] S. K. Kailasam, J. C. Lacombe, and M. E. Glicksman, "Evaluation of the methods for calculating the concentration-dependent diffusivity in binary systems," Metallurgical and Materials Transactions A, vol. 30, no. 10, pp. 2605-2610, 1999.

[6] A. G. Nikitin, S. V. Spichak, Y. S. Vedula, and A. G. Naumovets, "Symmetries and modelling functions for diffusion processes," Journal of Physics D: Applied Physics, vol. 42, no. 5, Article ID 055301, 2009.

[7] M. Vach and M. Svojtka, "Evaluation of molar volume effect for calculation of diffusion in binary systems," Metallurgical and Materials Transactions B, vol. 43, no. 6, pp. 1446-1453, 2012.

[8] I. Medved and R. Černý, "Model formulas for facilitating determination of concentration-dependent diffusion coefficients," Metals and Materials International, vol. 21, no. 5, pp. 907-912, 2015.

[9] I. Medved' and R. Černý, “Coupled water and salt transport in porous materials: rapid determination of a varying diffusion coefficient from experimental data," Transport in Porous Media, vol. 105, no. 3, pp. 597-610, 2014.

[10] J. Bear and Y. Bachmat, Introduction to Modelling of Transport Phenomena in Porous Media, vol. 4, Kluwer, Dordrecht, The Netherlands, 1990.

[11] C. Andrade, R. d'Andrea, and N. Rebolledo, "Chloride ion penetration in concrete: the reaction factor in the electrical resistivity model," Cement and Concrete Composites, vol. 47, pp. 41-46, 2014.

[12] Z. Pavlík, P. Michálek, M. Pavlíková, I. Kopecká, I. Maxová, and R. Černý, "Water and salt transport and storage properties of Mšené sandstone," Construction and Building Materials, vol. 22, no. 8, pp. 1736-1748, 2008.

[13] Z. Pavlík, L. Fiala, J. Maděra, M. Pavlíková, and R. Černý, "Computational modelling of coupled water and salt transport in porous materials using diffusion-advection model," Journal of the Franklin Institute, vol. 348, no. 7, pp. 1574-1587, 2011.

[14] H. S. Carslaw and J. C. Jaeger, Conduction of Heat in Solids, Clarendon Press, Oxford, UK, 1959.

[15] J. Crank, The Mathematics of Diffusion, Clarendon Press, Oxford, UK, 1975. 


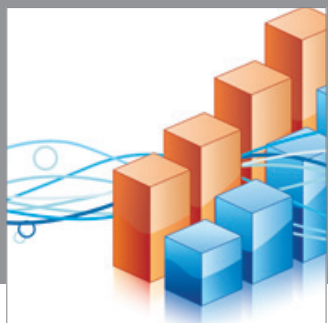

Advances in

Operations Research

mansans

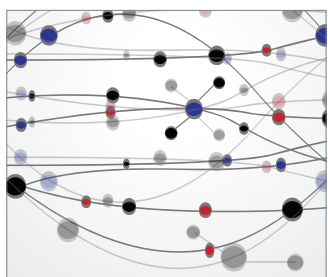

The Scientific World Journal
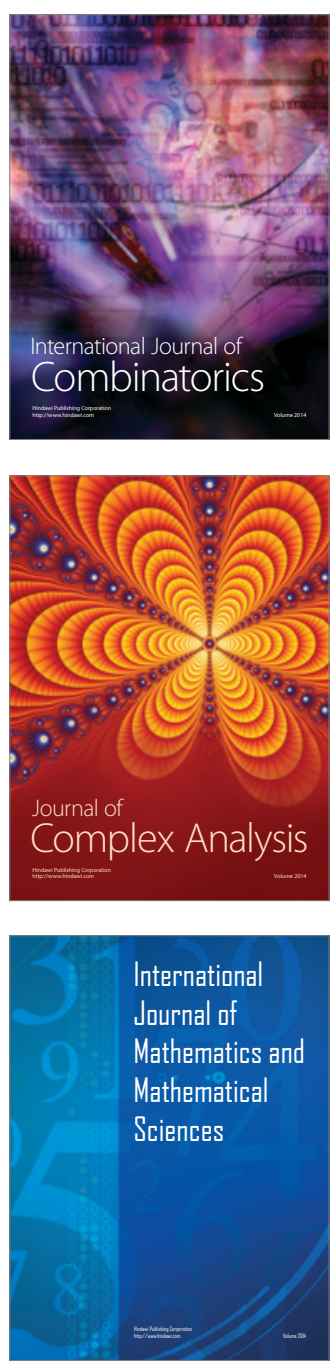
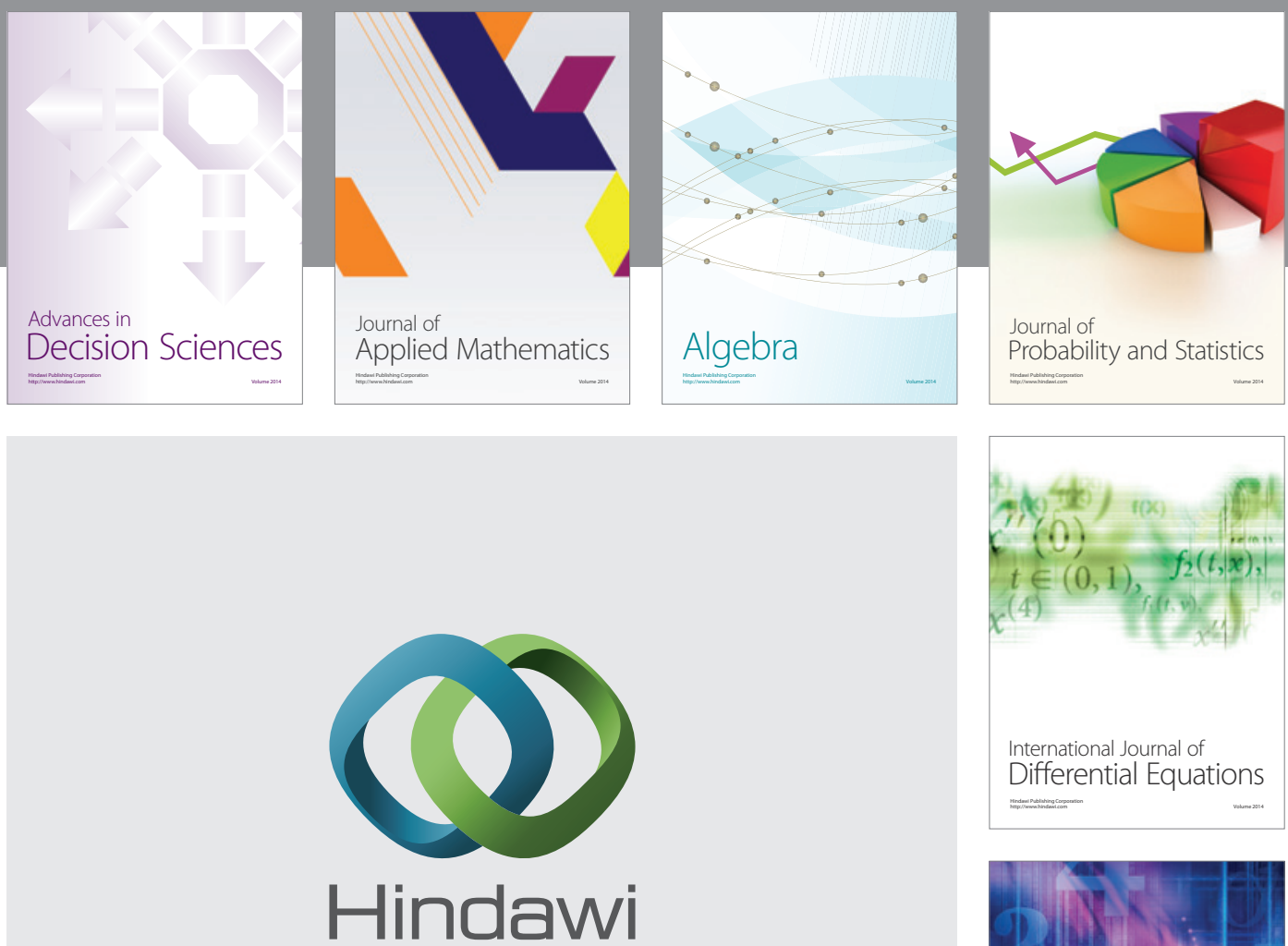

Submit your manuscripts at http://www.hindawi.com
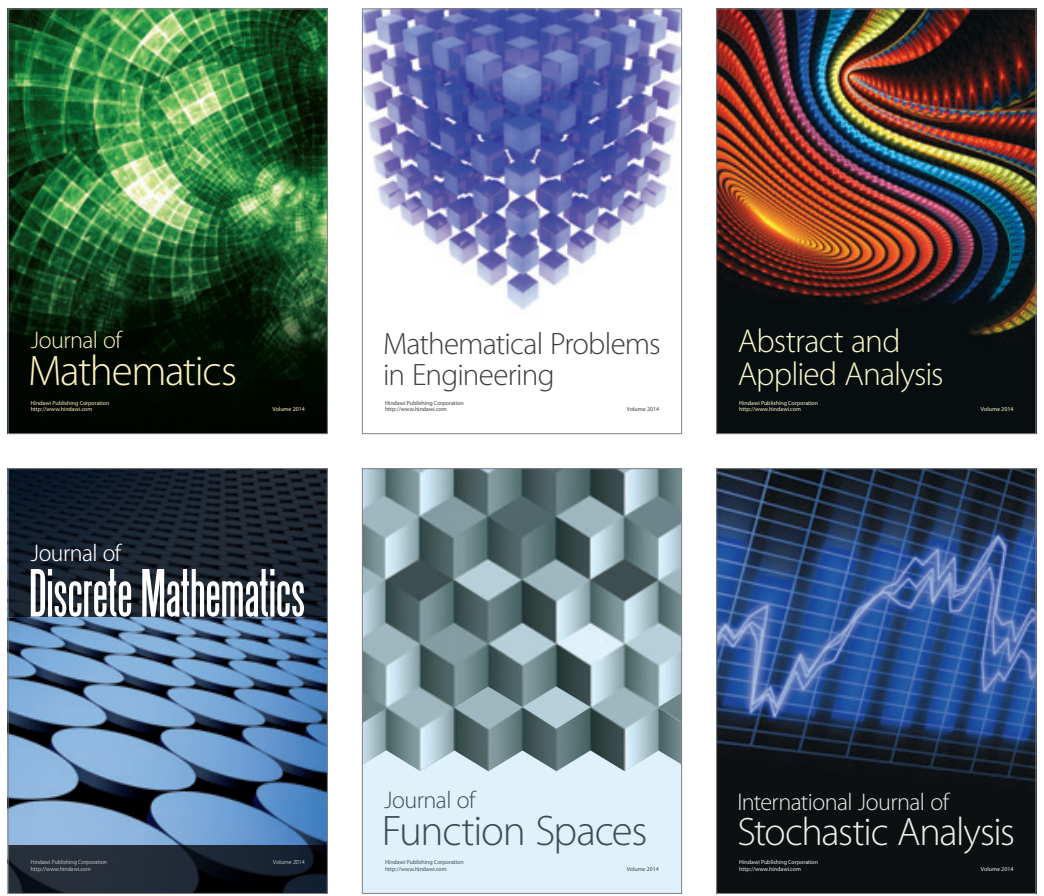

Journal of

Function Spaces

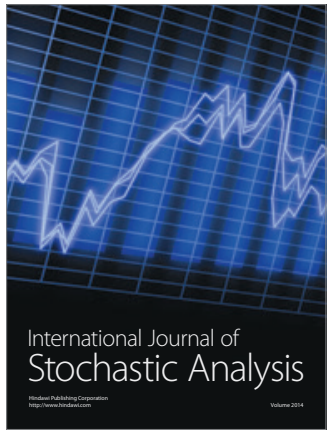

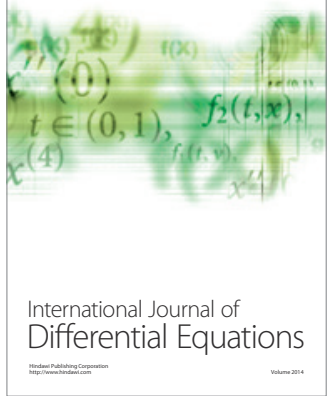
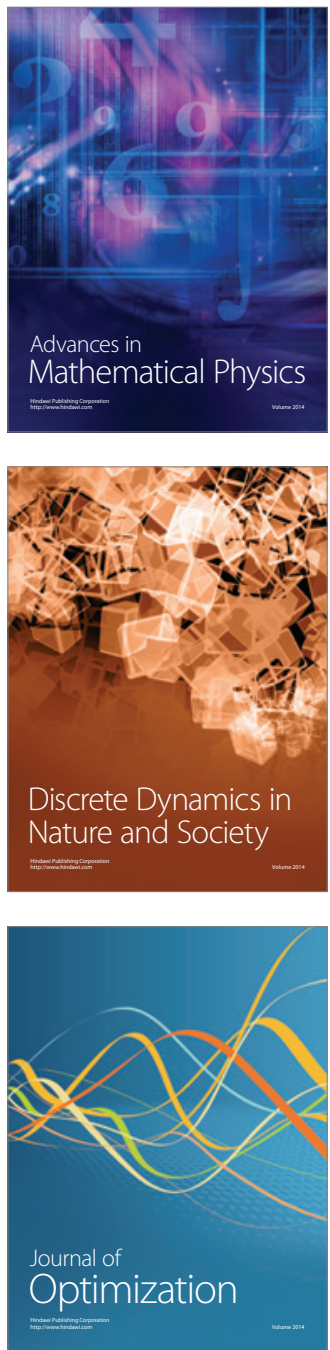